\title{
Transplant Co-ordination Services in Europe 1989: A Survey by the EDTA Registry
}

\author{
Data from the EDTA Registry \\ C. Bergstrom Transplantation Unit, University \\ Hospital, Uppsala, Sweden \\ G. Tufveson Transplantation Unit, University \\ Hospital, Uppsala, Sweden \\ M. Broyer Hôpital Necker Enfants-Malades, \\ Paris, France \\ F. P. Brunner* Department für Innere Medizin, \\ Universität Basel, Switzerland \\ H. Brynger Department of Surgery 1, Sahlgrenska \\ Sjukhuset, Göteborg, Sweden \\ J. H. H. Ehrich Medizinische Hochschule Hannover, \\ Federal Republic of Germany
}

\author{
W. Fassbinder Städtische Kliniken Fulda, Federal \\ Republic of Germany \\ W. Geerlings Stichting Thuisdialyse Noord \\ Nederland, Haren, The Netherlands \\ G. Rizzoni Ospedale Pediatrico Bambino Gesú, \\ Istituto di Ricerca Scientifica, Roma, \\ Italy \\ N. H. Selwood UK Transplant Service, Bristol, United \\ Kingdom \\ A. J. Wing \\ Kingdom
}

*Chairman

\begin{abstract}
This report contains EDTA Registry data concerning transplant co-ordination services. It is a summary of the results of a special questionnaire mailed in 1989 to all renal transplant centres known to the EDTA Registry in Europe and Mediterranean countries. The response rate to the questionnaire was $86 \%$. A transplant co-ordination service was available in more than $70 \%$ of the centres. The larger the transplant activity of a centre, the greater was the likelihood of a co-ordinator service being available. The educational background of the transplant co-ordinator was most commonly that of nurse. More than $70 \%$ of the centres had one or two transplant co-ordinators. A service shared by several centres was fairly common in the United Kingdom and France. The most important duties and activities appeared to be
\end{abstract}

Correspondence and offprint requests to: Professor F. P. Brunner, EDTA Registry, St Thomas' Hospital, London SEI 7EH. the organisation of transportation of donor teams and organs, giving feedback to the donor hospital's staff, and providing information to the public regarding organ donation.

Key words: Transplant co-ordinator; Transplantation

\section{Introduction}

Many transplant centres have recognised the need for a special transplant co-ordination service to solve the problems and logistics of kidney and multiorgan donation and transplantation. In its traditional role of collecting and comparing data on centre policy regarding all aspects of renal transplantation, the EDTA Registry designed a special questionnaire regarding transplant co-ordination. 
This was mailed early in 1989 to all transplant centres known in Europe and the Mediterranean countries. Centres were asked about the availability, the activities and the professional background of their transplant co-ordinators. 'Background' information such as the number of transplants performed in recent years was derived from the Registry's data files.

\section{Materials and Method}

All 217 centres that reported to the EDTA Registry as having performed at least five renal transplants in 1988, were asked the following questions:

1. (a) How many persons provide transplant co-ordinator services for your centre?

(b) Are their services shared with other transplant centres?

2. Are the transplant co-ordinator services available in your centre:

Less than normal office hours?

During normal office hours?

More than normal office hours but not 24 hours?

24-hour service?

3. Do(es) the transplant co-ordinator(s) participate in any of the following activities:

Organising waiting lists?

Contacting patients?

Organising transportation for donor team?

Usually present in operating theatre during donor operation?

Organising transportation of retrieved organ?

Follow-up contact with relatives of cadaveric donor?

Personal feed-back to staff of donor hospital?

Giving information about organ donation to hospital staff and/or public?

Other activities (specify if you wish)

4. (a) What is the professional background of your transplant co-ordinator(s)?

(b) Is transplant co-ordination their main activity?

5. From what year were transplant co-ordinator services available to your centre?

\section{Results}

Out of 187 replies, the availability of a transplant co-ordinator service was reported in $70 \%$ of centres. To achieve some demographic perspective, the data were analysed for selected countries or geographical regions. The results are displayed in Fig. 1. More than $70 \%$ of centres in the UK, France, Spain, FRG and the Benelux countries reported having a transplant co-ordinator service available.

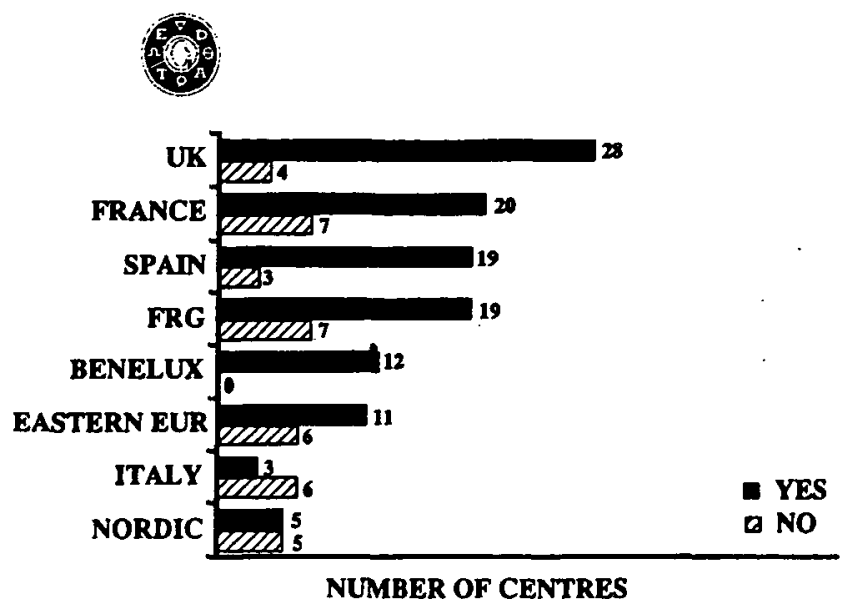

Fig. 1. Availability of a transplant co-ordination service in selected large countries or regions in 1989.

The year in which the service was started varied greatly between centres (Fig. 2). For a large proportion of about $40 \%$ of centres it was the 3-year period between 1984 and 1986. This was a period of increasing activity, not only in renal grafting, but more importantly in the use of multiple organ donation for rapidly expanding activity in heart, liver and pancreas transplantation. The centre activity, measured as the number of renal transplants performed during 1988 , had some correlation to the presence of a transplant co-ordinator service (Fig. 3). More commonly, the service was not shared between centres except in the United Kingdom and France, where this practice seems to be rather frequent. As can be seen from Fig. 3, the more active the transplant centre, the greater the likelihood of a transplant co-ordinator service. Background data for other transplantation activities such as heart, liver, and pancreas are unfortunately not available.

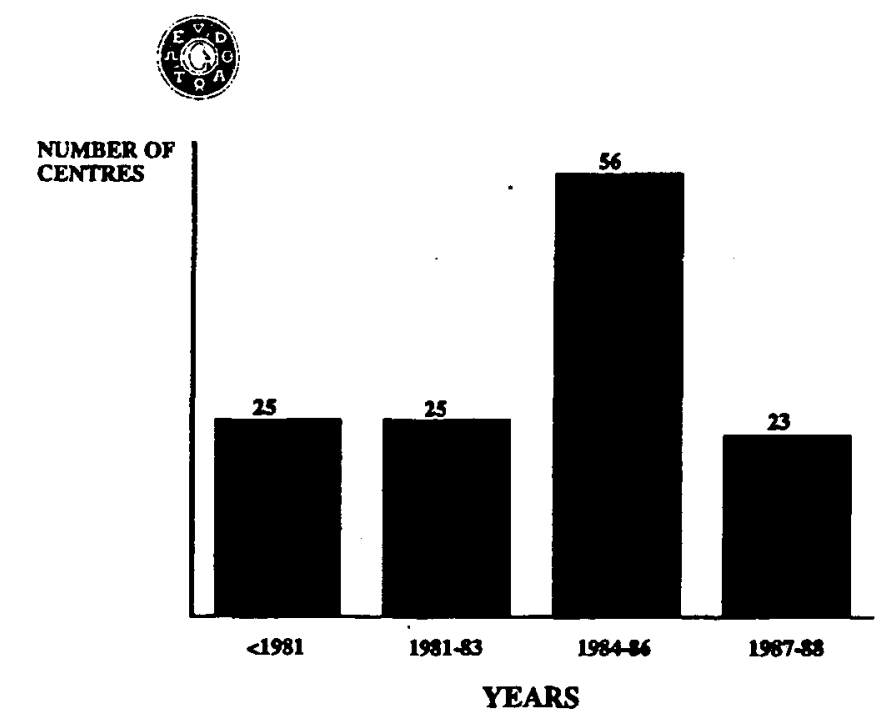

Fig. 2. The number of centres with a transplant co-ordination service according to the year of starting the service. Centres with shared co-ordination services are excluded. 


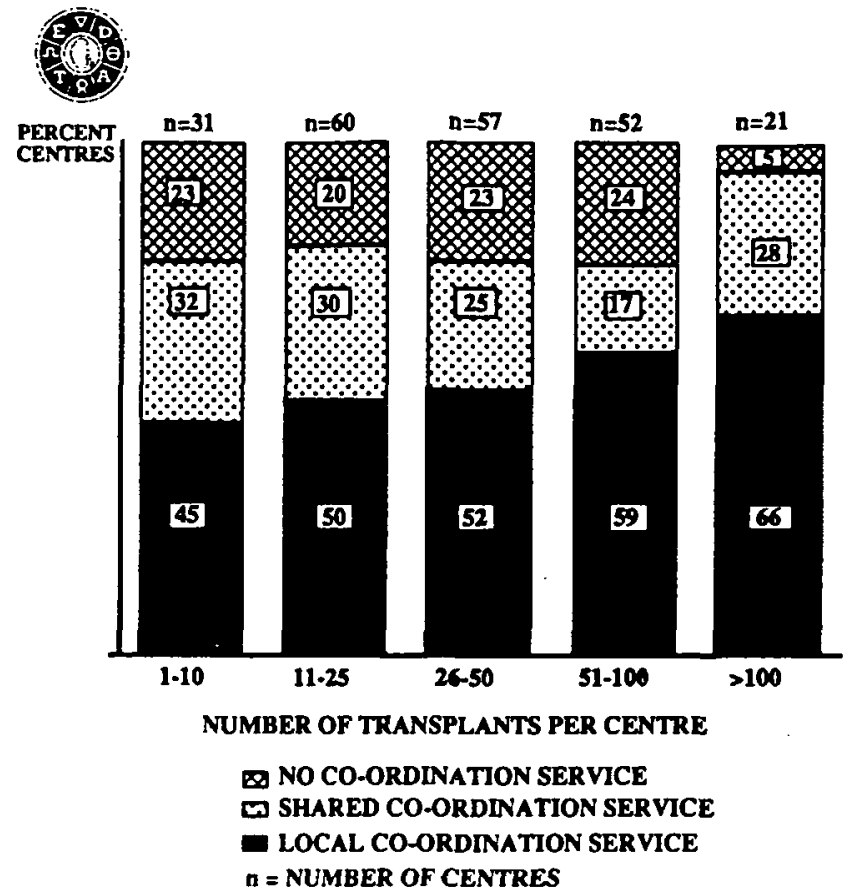

Fig. 3. Proportion of transplant centres reporting 'no co-ordination service', 'a shared co-ordination service', or 'a local co-ordination service', according to the transplant activity expressed as the number of renal transplants per centre in 1988. Since some centres reported both a shared as well as a local service; the number of centres given at the top of the bars exceeds the total number of questionnaires returned.

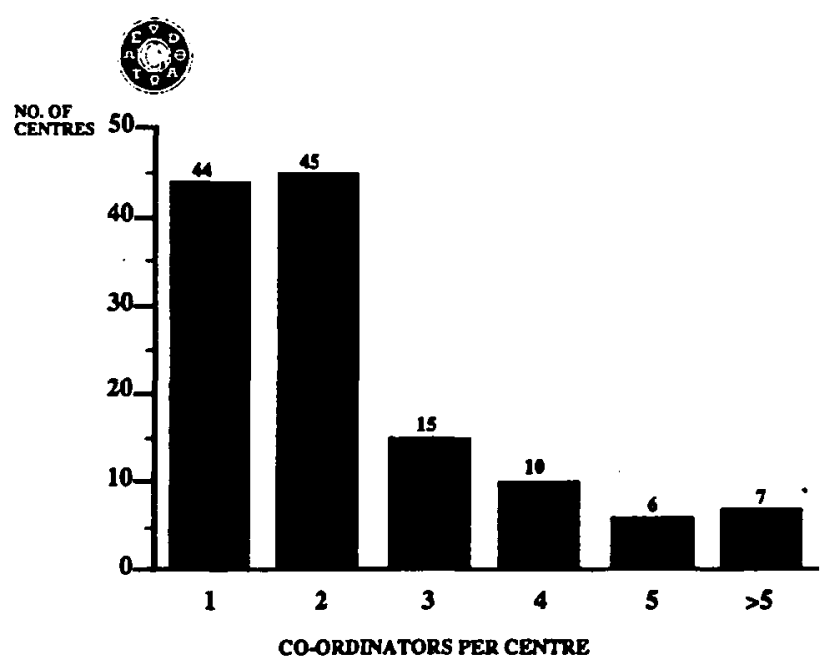

Fig. 4. Number of transplant co-ordinators per centre.

As depicted in Fig. 4, more than half of the transplant centres who replied had one or two transplant co-ordinators. A few centres, however, reported five or more transplant co-ordinators. A summary of the educational background of the full-time transplant co-ordinators is given in Fig. 5. The most common professional background was that of nurse, followed by doctor, medical

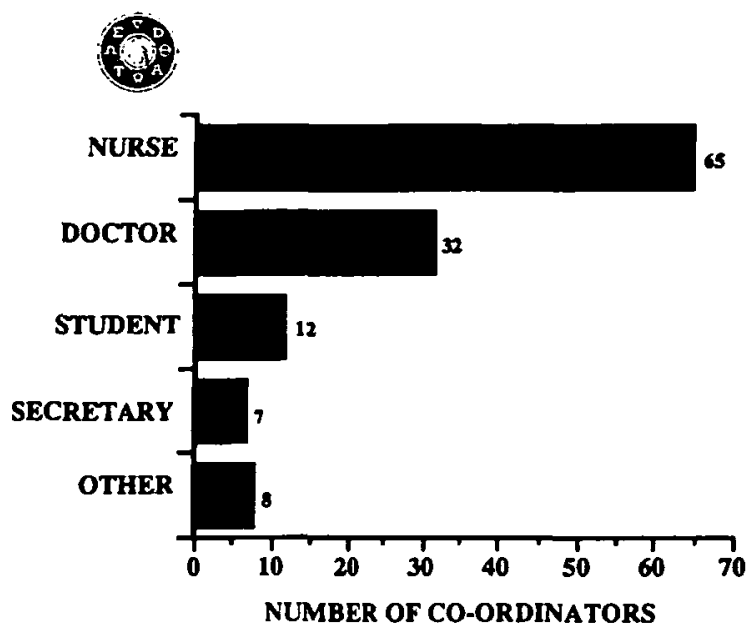

Fig. 5. The professional background of 124 full-time transplant coordinators.

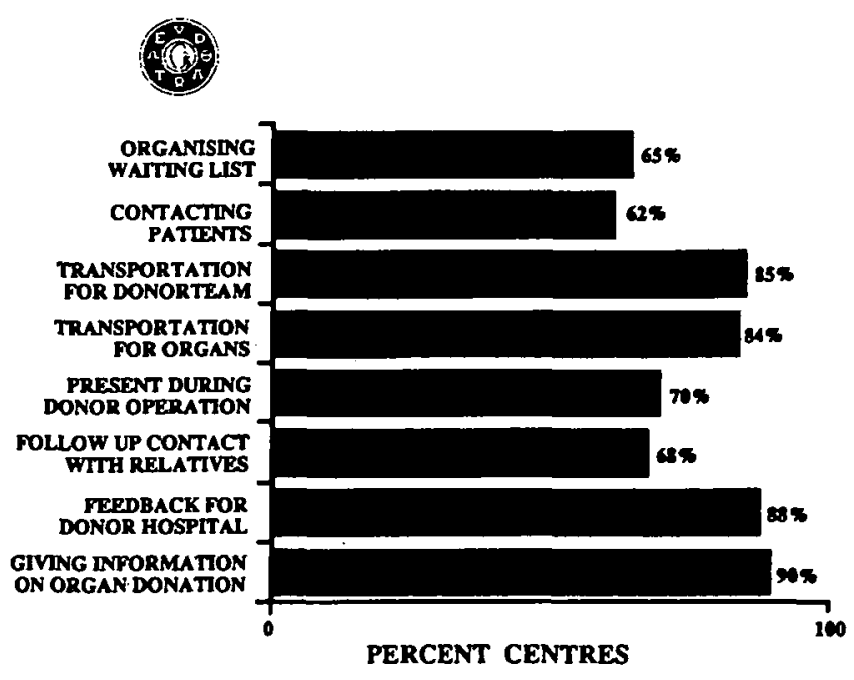

Fig. 6. The proportion of centres giving an affirmative answer to various duties and activities of their transplant co-ordinator(s).

student, secretary, and finally other. It seems likely that the nursing background of many co-ordinators may facilitate contact with donor hospital staff and thereby increase staff awareness and positive attitude towards organ donation.

Questions were also asked concerning the activities of the transplant co-ordinator. More than $90 \%$ of the centres reported that their transplant co-ordinators were giving information on organ donation to the public, and as many as $\mathbf{8 8 \%}$ supplied feedback information to donor hospitals and staff. Organisation of transportation for donor teams and transportation of organs also was important with more than $80 \%$ of the centres answering 'yes' for that activity. To a somewhat lesser extent, the co-ordinator was reported as actually being present during the donor operations (70\%). Activities like organising 
the waiting list and contacting patients on the waiting list, as well as follow-up contacts with donor relatives, were also reported to form an integral part of the task of the co-ordinator in more than $60 \%$ of the centres with a co-ordination service. The data are summarised in Fig. 6.

\section{Comment}

More than $70 \%$ of the centres replying have transplant co-ordinator services. The service was initiated most commonly between 1984 and 1986 . Well over $50 \%$ of the transplant co-ordinators are nurses and one-third are doctors. Of intrinsic interest is the $12 \%$ of medical students who are deemed to perform the function of transplant co-ordinator. More than $70 \%$ of the renal grafts are arranged with the help of transplant co-ordinators. The more active the centres, the greater the likelihood of a transplant co-ordinator. Taken together, it seems that transplant co-ordination should be regarded as having become an integral function within the transplant community. It may therefore be anticipated that this profession will further develop to meet the rising demands and assure the smooth running of the growing transplant programmes.

Acknowledgements. It is acknowledged that this study is general in its scope and lacks some detailed information that could be of interest. The high $(86 \%)$ response rate gives encouragement to look at some aspects in greater detail.

\section{Reference}

1. Brunner FP, Brynger H, Ehrich JHH et al. Combined report on regular dialysis and transplantation in Europe, XIX, 1988. Nephrol Dial Transplant 1989, 4 [Suppl 4]: 5-40

Received for publication 22.1.90

Accepted in revised form 3.5 .90 